\title{
Geschichte des öffentlichen Rechts in Deutschland
}

\author{
Michael Kloepfer*
}

Rezension zu Michael Stolleis, Geschichte des öffentlichen Rechts in Deutschland, Vierter Band: Staats- und Verwaltungsrechtswissenschaft in West und Ost 1945-1990, München (C. H. Beck) 2012, 720 Seiten, 68.- €. ISBN 978-3-406-63203-7

1. Nach 25 Jahren hat M. Stolleis (S.) nun mit seinem vierten Band - vom Kriegsende (1945) bis zur Wiedervereinigung (1990) - seine im Jahre 1600 einsetzende Darstellung der Geschichte des öffentlichen Rechts vollendet. Das fordert Respekt für die Leistung eines halben Berufslebens eines Professors und für seine ungewöhnliche wissenschaftliche Ausdauer.

In dem bisher umfangreichsten Band seiner Tetralogie versucht S. nach der Behandlung der Zeit von 1945-1990 noch mit einem „Rückblick“ und einem „Nachwort“ so etwas wie eine Betrachtung des Gesamtertrags seiner vier Bände. Das Gesamtwerk ist indessen nicht Gegenstand der vorliegenden Rezension, sondern nur der IV. Band. Dabei geht es im Folgenden um eine kompakte Schilderung des Inhalts (s. u. 2) und um eine Gesamtwürdigung (s.u. 3 u. 4) des Bandes.

2. Das Buch ist im Großen und Ganzen - aber gewiss nicht streng - chronologisch gegliedert. Es ist zeitlich eher schindelartig angeordnet, sodass viele Themen (bspw. die Studentenunruhen) mehrfach im Buch angesprochen werden. Das führt zu nicht wenigen Redundanzen. Dieser zeitliche Überlappungseffekt der Schindel-Bauweise wird noch dadurch verstärkt, dass S. häufig einzelne Sachprobleme über engere Zeitgrenzen hinaus erörtert. Bisweilen fällt es dem Leser schwer, den jeweiligen Zeitraum exakt zu bestimmen, auf den sich eine bestimmte Bemerkung des Buches bezieht.

Der Schwerpunkt der Darstellung liegt auf der bundesrechtlichen Entwicklung, gleichfalls wird aber auch die Entwicklung in der DDR behandelt. Diese an sich gute Idee wird jedoch nur bedingt erfolgreich verwirklicht. Die Entwicklungsphasen der DDR werden den jeweiligen Zeiträumen der bundesdeutschen Entwicklung zugeordnet, ohne dass - außer vielleicht in der Anfangssituation und bei der Wiedervereinigung - eine hinreichende inhaltliche Verzahnung zwischen der ost- und der westdeutschen Entwicklung erfolgt. So verbleiben etwa die Bezüge von „Babelsberg“ (S. 289 ff.) zur Wirtschaftswunderzeit im Westen im Dunkeln. S. weist selbst auf die „anderen Zäsuren“ der Entwicklung in der DDR hin. Insgesamt geht das Buch trotz seines Titels weit über 1990 hinaus, selbst Fukushima (2011) wird noch erwähnt.

a) Im ersten Kapitel (Wiederaufbau und Selbstfindung) werden zunächst die Forschungslage und die Methodik erörtert, deren Darlegung an sich besser in eine Einleitung gehört hätte. Es folgen - für die Zeit von 1945 bis zum Anfang der 1950er

* Prof. em. Dr. Michael Kloepfer, Juristische Fakultät der Humboldt-Universität zu Berlin. 
Jahre - eindrückliche Darlegungen zur „Stunde Null“ mit der grundsätzlich sehr berechtigten Kritik an der Übernahme von NS-Belasteten in die Macht- und Wirtschaftsstrukturen des frühen Westdeutschlands. Die Einbindung von Funktionsträgern der DDR nach der Wiedervereinigung in den gesamtdeutschen Staat wird von S. deutlich nachsichtiger beurteilt. Sodann folgen eher lexikalische Darstellungen des personellen Wiederaufbaus der westlichen Universitäten, d.h. genauer der juristischen Fakultäten, wobei viele Kurzcharakterisierungen der behandelten Personen treffend sind. Anschließend erfolgt ein Überblick über die Neu- und Wiedergründungen juristischer Gesellschaften einschließlich der Staatsrechtslehrervereinigung sowie über die juristischen Zeitschriften, wobei bedauerlicherweise „Der Staat“ keinen eigenen Abschnitt erhalten hat. Die Darlegungen zur „SBZ und erste Jahre der DDR “ bringen wieder lexikalische Schilderungen der im Osten verbliebenen Fakultäten und der dortigen außeruniversitären Institutionen. Hier wäre es interessant gewesen, etwas Konkreteres über den Einfluss der sowjetischen Besatzungsmacht, aber auch der SED und der Staatssicherheit auf die ostdeutschen Universitäten und Wissenschaftsinstitutionen zu erfahren.

Die Zwangsvereinigung von SPD und KPD wird - wenn ich nichts überlesen habe erstaunlicherweise nicht erwähnt. Eine gewisse terminologische Lässigkeit von S. fällt auf: Es wird die frühere SED-Schreibweise „Westberlin“ übernommen, vereinzelt wird - DDR-mäßig - auch von „BRD“ gesprochen (S. 603).

Bedauerlich ist, dass S. kein Wort zu den ehemals deutschen Fakultäten im heutigen Polen verliert. Hier wäre gewiss noch manches zu erforschen, insbesondere zu den Schicksalen der früher dort tätigen deutschen Professoren und zu dem Verbleib der Fakultätsbibliotheken etc. Wenig einleuchtend ist - gerade im geschichtlichen Kontext des Gesamtwerkes - die völlige Ausblendung des Schicksals der österreichischen Fakultäten nach 1945. Mancher Vergleich zwischen der österreichischen und der deutschen Entwicklung wäre hier sicherlich interessant gewesen.

b) Das zweite Kapitel (Grundgesetz und Staatsrechtslehre) behandelt schwerpunktmäßig die Zeit von 1946-1965 und befasst sich zunächst mit der Entstehung der West-Länder und des Grundgesetzes sowie mit den anfänglichen Veröffentlichungen zum öffentlichen Recht, insbesondere mit den damaligen Lehrbüchern und Kommentierungen zum Grundgesetz.

Es folgen hellsichtige Betrachtungen zum BVerfG (S.145 ff.), wobei dessen erste Richtergeneration zutreffend (auch) in ihrer großen Distanz zum Nationalsozialismus vorgestellt wird. Die diskursbeherrschende Rolle des Gerichts wird - gerade wegen seiner Standfestigkeit gegenüber der Politik - betont, wobei einige wichtige Entscheidungen aus der Frühzeit des Gerichts vorgeführt werden. Es folgt eine Darstellung der Lage des westdeutschen Verwaltungsrechts wiederum im Wesentlichen 
anhand bewertender Darstellungen der damals vorliegenden Lehrbücher. Der Neuaufbau der westdeutschen Verwaltungsgerichtsbarkeit einschließlich der Sozialgerichtsbarkeit wird geschildert. Auf die Verwaltungswissenschaft geht S. ebenso ein wie auf die - schon damals an Bedeutung verlierende - Allgemeine Staatslehre und auf das Völkerrecht. Dabei wird wieder auf die Lehrbuchliteratur besonderes Gewicht gelegt, während die völkerrechtliche Praxis fast ganz in den Hintergrund gerückt wird.

c) Das dritte Kapitel befasst sich - in starker zeitlicher Überschneidung mit dem zweiten Kapitel - mit dem „Rechtsstaat und Sozialstaat im ,Wirtschafswunder'“. Es folgen kurze grundsatzhafte Ausführungen zum Rechtsstaat, zum Rechtsschutz, zu den Grundrechten und zur Rechtsprechung des BVerfG, zur Abtreibungsproblematik und zu den besonderen Gewaltverhältnissen. Die Lüth-Entscheidung wird innerhalb von 60 Seiten zweimal behandelt (S. 165, 225 ff.), wobei S. vor allem auf die Kritik von C. Schmitt und E. Forsthoff eingeht. Zu einfach macht er sich die Darstellung des Wirtschaftsverwaltungsrechts (S. 236 ff.), die im Wesentlichen auf die Berichte über thematisch einschlägige Staatsrechtslehrertagungen reduziert und kurze Zeit später durch eine Darstellung des öffentlichen Wirtschaftsrechts ergänzt wird. Beides hätte zusammengehört. Unterkomplex (aber nicht falsch) ist die simple Auflistung wichtiger Ordnungsrechts- und Verfassungsprobleme (S. 240 f.). Das Bild der Verfassung unter dem Grundgesetz als „Zivilreligion“ (S. 242, 245) ist nicht ganz neu.

S. befasst sich sodann mit den Auswirkungen auf das Verwaltungsrecht, wobei er die Rolle der Grundrechte überzeichnet und merkwürdigerweise auf die kurz vorher erörterte Konstitutionalisierung der Rechtsordnung nicht eingeht, dafür aber auf das Schlagwort F. Werners vom Verwaltungsrecht als konkretisiertes Verfassungsrecht. Wieder werden die damaligen einschlägigen Staatsrechtslehrertagungen behandelt, die freilich die vielfältigen konkreten Entwicklungen des Verwaltungsrechts nur unvollständig abbilden konnten. Es folgen Überlegungen und Darstellungen zum System des Verwaltungsrechts und zur Kodifikation des Verwaltungsverfahrensrechts. Auf die zentrifugalen Bewegungen durch die vielen Sondergebiete des Besonderen Verwaltungsrechts wird eingegangen. Insbesondere werden das Planungsrecht, das öffentliche Wirtschaftsrecht (wobei S. im Wesentlichen nur die Fragen der Wirtschaftsverfassung betrachtet) sowie das Bildungsrecht und das Verwaltungsprozessrecht kurz behandelt.

Die Ausführungen zum Sozialstaat und zur Entwicklung des Sozialrechts kritisieren zunächst die verfassungsrechtliche Regelungszurückhaltung zum Sozialstaat und breiten bei den Passagen zur Entwicklung des Sozialstaates im Wesentlichen die bekannte damalige Diskussion über die rechtliche Bindungskraft des Sozialstaatsprin- 
zips aus. Es folgen Darstellungen zur Entwicklung der Sozialgerichtsbarkeit und des Sozialrechts.

In eigentümlicher Gliederung - S. vergibt keinen Gliederungspunkt - als Unterpassage des dritten Kapitels tauchen dann an sich recht interessante Bemerkungen zu „Babelsberg und den Folgen in der DDR“ auf. Was das mit den gleichzeitigen westdeutschen Entwicklungen zu tun haben soll, bleibt freilich offen. Auf Drängen der SED und Ulbrichts und unter Mithilfe von Polak verschwand damals bekannterweise für die nächsten zwölf Jahre in der DDR das Verwaltungsrecht. S. gelingt es dabei allerdings nicht, „Babelsberg“ historisch hinreichend zu erklären. Die Gründe diese Abschaffung des Verwaltungsrechts, werden kaum deutlich. Das Staatsrecht in der DDR wurde indessen (auf m.E. freilich meist dürftigem Niveau) weiterbetrieben. Für „Babelsberg“ gilt also in Umkehrung zu Otto Meyer: Verwaltungsrecht vergeht, Verfassungsrecht besteht. Aus dem Verwaltungsrecht wurde ein „Leitungsrecht“.

d) Das vierte Kapitel „Lehrjahre der Demokratie“ geht von den Entwicklungen der fünfziger und sechziger Jahre (bis 1968) aus und verzichtet in diesem Kapitel völlig auf die Darstellung zeitgleicher Entwicklungen in der DDR. S. berichtet hier von den Schwierigkeiten in Westdeutschland, demokratische Strukturen innergesellschaftlich durchzusetzen und erörtert insbesondere die wehrhafte Demokratie (mit den Parteiverboten, der Grundrechtsverwirkung und der Nichtzulassung von Extremisten zum öffentlichen Dienst).

Unter dem Titel „Demokratie und Gesellschaft“ schildert S. vornehmlich die Ablösung der CDU-geprägten konservativen Herrschaftsstrukturen durch die sozialliberalen Mehrheiten in Westdeutschland und die sich daraus ergebende Umbruchsituationen einschließlich der grundlegenden Veränderungen in der damaligen Zeit sowie die Enstehung der APO und die Studentenrevolte. Bezüglich der Notstandsdebatte in der Bundesrepublik Deutschland kommt der Aspekt des deutschen Souveränitätsrückgewinns durch die Notstandsgesetze etwas zu kurz. Im Bezug auf die Studentenrevolte verschweigt $\mathrm{S}$. deren teilweise totalitären Auswüchse nicht, wohl aber die damalige Kultur des „Wegsehens“ bei vielen professoralen Kollegen gegenüber solchen Auswüchsen (letztlich nach dem St. Florians-Prinzip).

Es folgen eher essayistische Bemerkungen zu den politischen Parteien, zu den Bürgerinitiativen und zur Arbeitnehmermitbestimmung in Unternehmen. Auch die Kirchen werden behandelt und zwar in sachlich ungerechtfertigter Frageform: „Freie Kirche im demokratischen Staat?" Diese Erörterungen reichen bis hin zur Zukunft des Staatskirchenrechts angesichts des Vordringens des Islams in Deutschland. Der grundsätzlich gerechtfertigten kritischen Sicht zur Rolle der Kirche im NS-Staat stellt S. keine entsprechende Darstellung zur bisher nur eingeschränkt erforschten Rolle der Kirchen in der DDR gegenüber. 
Wiederum zeitlich überlappend und wiederholend wendet sich S. dann der „Staatsrechtslehre und Demokratie nach 1968“ mit der wissenschaftlichen Diskussion um das parlamentarische Regierungssystem zu und kritisiert die weitgehende Angepasstheit der nach S. seit jeher - angeblich - ausgeprägt konservativen Staatslehre an die herrschende Politik. S. beschreibt sodann das damalige weitgehende Vakuum hinsichtlich einer neuen Staatstheorie.

Dabei überschätzt er allerdings die im Buch mehrfach beschriebene Kontroverse von C. Schmitt und R. Smend für die Zeit nach 1968. Schon damals war die Schilderung dieser Diskussion doch bereits zu einem guten Teil bloße akademische Brauchtumspflege. S. öffnet sich auch den US-amerikanischen Einflüssen durch die ehemaligen Emigranten. Es folgen wiederum Quasi-Kurzrezensionen zu den damals verbreitetesten Lehrbüchern zur Allgemeinen Staatslehre und die Schilderung systemkritischer Stimmen insbesondere von „linker“ Seite sowie die Erwähnung neuer rechtstheoretischer Ansätze, die immerhin bei der Gründung des Grundlagen-Arbeitskreises bei der Staatsrechtslehrertagung im Jahr 2010 endet.

e) Unter nochmaliger zeitlicher Überlappung mit dem bereits vorher Ausgeführten, wendet sich S. im fünften Kapitel dem Thema „Unruhe, Expansion, Neuordnung“ zu. Es tauchen also wieder APO, Studentenrevolte und ihre Ursachen auf, der Umweltschutz hingegen kaum. Die Kennzeichnung von Dutschke durch S. als „Rednerguru“ ist nicht wissenschaftlich. Der als Gegenbewegung zur Studentenrevolte gegründte „Bund Freiheit der Wissenschaft“ bestand im Übrigen zu erheblichen Teilen aus aktiven bzw. ehemaligen Sozialdemokraten.

Auch die Ausführungen „Vom Eingriffs- zum Leistungs- und Planungsstaat“ wiederholen ebenfalls weitgehend bereits im Buch schon Geschriebenes. Die Universitätsneugründungen werden vor allem in ihrer personellen Zusammensetzung ebenso geschildert wie - relativ teilnahmslos - die Reformen der Juristenausbildung. Bei der Vorstellung der damaligen westlichen Fakultäten wird z.B. vernachlässigt, dass an der Juristischen Fakultät der FU Berlin zunächst die „Linken“ durchaus das Sagen hatten und bei Bremen wird G. Winter einfach ebenso übersehen wie der Umstand, dass nun dort wohl aus Prinzip keine Habilitierten berufen wurden.

Unter dem Titel „Staatsrechts- und Verwaltungsrechtslehre nach 1968“ schildert S. nun Veränderungen der sozialen Befindlichkeiten in der damaligen Gesellschaft und das Eindringen der Sozialwissenschaften in die Wissenschaft des öffentlichen Rechts. Es tauchen erneut der Club of Rome und der Ölschock etc. auf. Das Problem der Großprojekte und ihrer gerichtlichen Kontrolle wird gestreift.

Erneut geht S. auf die Verwaltungslehre und auf einige Staatsrechtslehrertagungen der damaligen Zeit ein. Anschließend wird die „Neue Ostpolitik“ der Bundesrepublik Deutschland nebst den Reaktionen der Staats- und Völkerrechtler geschildert. 
Dem folgen Kurzüberblicke über die damals gebräuchlichen Staatsrechtslehrbücher (P. Baduras Werk fehlt) und Zeitschriften.

f) Das sechste Kapitel „Konsolidierung und Krisenmanagement“ befasst sich explizit mit den Jahren von 1972-1990. Erneut kommt es zu Wiederholungen. Der Club of Rome und der Ölschock erscheinen ein weiteres Mal. Die Nachrüstungsdebatte wird geschildert. Es folgen Darstellungen von Staatsrechtslehrertagungen in dieser Zeit. Überzeichnend ist die Behauptung von S., „die Grünen“ würden im Kern konservative Ziele verfolgen (S. 505). Im Nachhinein folgen etwas bunt zusammengestellte Betrachtungen zu Leistungsrechten der Bürger und zu Schutzpflichten des Staates, zu den Grundpflichten des Bürgers und zu den organisierten Einwirkungen auf die Verwaltung sowie zu den neuen verwaltungsrechtlichen Steuerungsformen. Das verstärkte Eindringen der Sozialwissenschaften in das Verwaltungsrecht habe gelegentlich zu einem „affektierten Neusprech“ geführt (S. 514). Schließlich wird noch einiges zur Gleichberechtigung (bis hin zu „Emma“) gesagt. Es folgen Äußerungen zum Allgemeinen Teil des Verwaltungsrechts und zu den modernen Teilgebieten des Verwaltungsrechts (Denkmalschutz, Umweltschutz, Telekommunikations- und Technikrecht), wobei die EG/EU-Einflüsse und besonders der Einfluss der USA praktisch ausgeblendet bleiben. Die teilweise auf wenige Zeilen beschränkten Darstellungen erreichen überwiegend kein substanzielles Niveau.

Richtig ist die Beobachtung von S., dass die Grenzen zwischen öffentlichem und privatem Recht zunehmend verwischen. Beobachtungen zur „Opulenz der (öffentlichrechtlichen) Literatur" schließen sich an und geraten teilweise zu einem freilich sehr kurz gefassten Literaturführer. Dabei bleibt allerdings die Literatur zum Besonderen Verwaltungsrecht weitestgehend ausgespart. S. geht auf den Konzentrationsprozess der juristischen Verlage in Westdeutschland (mit dem drohenden Vielfaltsverlust) leider nicht ein.

S. wendet sich sodann dem Staats-und Verwaltungsrecht in der Endphase Ulbrichts und in der Honecker-Zeit zu. Dabei kommt der Einfluss von Gorbatschow und seiner Politik insgesamt viel zu kurz. Man erfährt Einiges über die regelmäßig von Autorenkollektiven verfassten Lehrbücher der DDR (zum Staatsrecht, zum Staatsrecht der bürgerlichen Staaten, zum Verwaltungsrecht und zum Völkerrecht) sowie über die juristischen Fachzeitschriften der DDR. Auch die Ausführungen über die A- und B-Dissertationen sowie über das Qualifikations- und Auszeichnungssystems der DDR bergen Interessantes. Stärker herausgestellt hätte der erdrückende Einfluss der SED (auch) auf das wissenschaftliche Publikationswesen der DDR werden müssen. Die Personalien der vier verbliebenen juristischen Fakultäten des Ostens werden vorgestellt. Dabei hätte man gerne noch etwas Näheres über die Karrierebedingungen der DDR-Forscher und über den Einfluss der SED sowie der Staatssicherheit auf die juristischen Fakultäten erfahren. Instruktiv sind auch die Ausfüh- 
rungen zur (Deutschen) Akademie für Staats-und Rechtswissenschaft ((D)ASR) in Babelsberg. Die Verbindungen der Juristischen Fakultäten der DDR zur Akademie der Wissenschaften der DDR bleiben unerörtert. Die juristische Hochschule des Ministeriums für Staatssicherheit in Golm wird kurz dargestellt.

g) Das siebte Kapitel steht unter dem Titel „Europäisierung und Wiedervereinigung “ und greift (bezüglich Europa) sogar auf die Zeit vor 1945 zurück. Im Hinblick auf die deutsche Frage wird im Wesentlichen die Zeit zwischen Grundlagenvertrag und Wiedervereinigung erörtert. Hinsichtlich Europas wird zunächst auf die „Westbindung der BRD“ eingegangen, wobei die trennungsvertiefenden Konsequenzen der Westbindung nicht problematisiert werden. Es folgen ein schulbuchartiger Geschwinddurchgang durch die Geschichte der Europäischen Integration und eine Darstellung zur Entwicklung des Europarechts als Fach. S. geht hier im Wesentlichen vom europäischen Primärrecht aus, während das europäische Sekundärrecht ausgeblendet bleibt. Unerörtert bleibt die Frage, ob das Fach Europarecht wirklich noch eine Zukunft hat, wenn dereinst praktisch alle Rechtsgebiete europarechtlich durchdrungen sein werden. Möglicherweise wird langfristig nur das Europäische Verfassungsrecht (oder Ähnliches) übrig bleiben. Vielleicht wird das Europarecht also das Schicksal des Faches „Reichsrecht“ in der ehemaligen Straßburger Fakultät im ausgehenden 19. Jahrhundert erleiden. Ein Fach „Bundesrecht“ erscheint uns jedenfalls heute zu Recht absurd.

Es folgen kurze, eher unterkomplexe Bemerkungen zum Problem einer Europäischen Verfassung. Die (deutschsprachigen) europarechtlichen Lehrbücher, Kommentare und Zeitschriften werden kurz vorgestellt. Praktisch nicht mehr erfasst werden konnte die derzeitige Euro-Krise, deren Bewältigungsversuche letztlich aber zu der Frage führt, ob die Idee Europas als Rechtsgemeinschaft hierdurch nicht substantiellen Schaden genommen hat.

S. erörtert den Wandel des Völkerrechts z.B. durch neue Akteure (bspw. NGOs) oder durch die - völkerrechtliche - Regionalisierung. Dabei hätte man sich noch einige Bemerkungen zum Aufbau der völkerrechtlichen Strafgerichtsbarkeit gewünscht. Wichtig ist der Hinweis auf den Einfluss der Nachbarwissenschaften auf das Völkerrecht.

Schließlich wendet sich S. der „Wiedervereinigung und (dem) Neuaufbau“ zu und geht dabei sinnvollerweise weit über das Jahr 1990 hinaus. Der Zerfall der DDR wird sehr kurz geschildert. Der Einfluss der einschlägigen Staatsrechtslehrertagungen auf die juristische Bewältigung der Wiedervereinigung wird gewiss überbewertet, der Staatsvertrag und der Einigungsvertrag zwischen beiden deutschen Staaten nebst den wiedervereinigungsermöglichenden und wiedervereinigungsbedingten Grundgesetzänderungen (Gemeinsame Verfassungskommission) werden vernachlässigt. 
Die Abwicklung der DDR wird von S. als weitgehend alternativlos dargestellt. Trotzdem urteilt S. kritisch: „Es war offenkundig, dass nun das stärkere Kollektiv über das schwächere urteilte“ (S. 646). Das schöpft das Problem nicht nur nicht aus, sondern stellt die Übernahme- bzw. Abwicklungsfragen schief dar. Die Nichtübernahme des bisherigen wissenschaftlichen Personals der DDR hatte eben nicht nur politische, sondern regelmäßig gerade auch qualifikationsbezogene Gründe. Auf das Problem der Entlastung der DDR-Juristen durch die fast völlige Konzentration der Entscheidungen über die Übernahme von Personal auf Stasiverstrickungen wird nicht eingegangen. Es folgen erneut personalbezogene Schilderungen der juristischen Fakultäten der neuen Bundesländer (einschließlich HU), wobei P. M. Huber in Jena übersehen wurde.

Es folgen dann - mit dem Pathos der Zukunftssuche - kursorische und überwiegend eher journalistische Bemerkungen zur weiteren Europäischen Entwicklung, zu Auslandseinsätzen der Bundeswehr, zu „9/11“, zum weiteren Ausbau der Europäischen Union, zur Globalisierung des Rechts, zu neuen Kriegsformen und zum Schutz der natürlichen Ressourcen sowie zur elektronischen Vernetzung der Welt (aber nicht zur Energiewende). Hier zeigt sich, dass der Prophetenmantel für S. doch wohl noch recht weit ist. S. fordert eine Durchdringung der „global governance“ mit demokratischen und menschenrechtlichen Gehalten. Zivilgesellschaft und Staat würden sich immer stärker durchdringen; künftig würden sich vermehrt offene government-Formen bilden. Auch bei einer allmählichen Ablösung des Staates werde das öffentliche Recht als solches möglicherweise zwar verschwinden, seine Sachregelungen würden aber bestehen bleiben, wenn auch in veränderter Form.

In einem „Rückblick“ erfolgt schließlich eine - mit Danksagungen angereicherte Rückschau auf das Gesamtwerk und auf die hierbei leitende Methodik. Ambitionierte, aber nicht immer klare Überlegungen zu einem „ius cummune publicum “ und zu den etwaigen Charakteristika eines „deutschen“ öffentlichen Rechts schließen sich an. Schließlich werden die vielen, ohnehin allgemeinen Beobachtungen des Buches nochmals verallgemeinert. Interessant, aber unbewiesen, bleibt die Abschlussbemerkung über die Ähnlichkeit prä- und postmoderner Strukturen. Der Band schließt mit einem „Nachwort“, das üblicherweise sonst als Vorwort dienen würde: ein Hauch Baselitz?

3. Das Gesamturteil über das - stilistisch regelmäßig ansprechend geschriebene und gut lesbare - Werk von S. muss ambivalent ausfallen, obwohl es schon zweimal in der FAZ besprochen wurde. Einerseits ist Respekt vor dem Gesamtwerk und vor der Pionierarbeit sowie vor der umfassenden rechtshistorischen Bildung von S. geboten. Ich habe bei der Lektüre des Buches sehr viel gelernt. Das Buch ist ein eindrucksvolles Zeugnis für die geistige Orientierung durch die Beschäftigung mit Geschichte. 
Andererseits sind aber erhebliche Schwächen im Gesamtkonzept, aber auch im „Finish“ des Buches unverkennbar. Die Ansprüche an das Buch sind (zu) vielfältig: es legt - wie schon der Titel zeigt - nicht nur eine Geschichte der Wissenschaft des öffentlichen Rechts vor, sondern ebenfalls eine Geschichte des öffentlichen Rechts als solches, was dann auch eine Darstellung der Entwicklung der Gesetzgebung und der Rechtsprechung des öffentlichen Rechts zwischen 1945 und 1990 fordert. Dies wird dann sinnvollerweise noch in Zusammenhänge der allgemeinen deutschen und internationalen Politik bzw. Geschichte der Berichtszeit gestellt. Das alles - allgemeine Geschichte, Geschichte des öffentlichen Rechts, Geschichte der Wissenschaft vom öffentlichen Recht in der Zeit von 1945 bis 1990 - lässt sich eben nicht mit hinreichender wissenschaftlicher Präzision auf 700 Seiten darstellen. Hinzu kommt, dass S. auch noch die Verwaltungslehre, die Allgemeine Staatslehre und das Völkerrecht sowie die Entwicklung in der DDR mit in seine Darstellung einbezieht. Zudem will S. noch etwas zur Zukunft des öffentlichen Rechts und des Staates sagen. Das alles überfordert das Buch erkennbar und führt nicht selten zu unterkomplexen Erörterungen teilweise eben nur auf Zeitungsniveau. Hinsichtlich der Thematik wäre gewiss weniger mehr gewesen. Unklar ist auch der Adressatenkreis, an den das Buch sich wendet. Manches scheint für die Juraprofessoren geschrieben (z.B. die Personalgeschichte der Fakultäten), vieles aber auch für den juristischen Laien (etwa die partiellen Einblicke in die Rechtsprechung des Bundesverfassungsgerichts).

So begrüßenswert die Idee einer „gesamtdeutschen“ Darstellung der Zeit von 1945 bis 1990 erscheint, kann das Nebeneinander der Darlegung der Entwicklung in der Bundesrepublik Deutschland einerseits und in der DDR andererseits kaum überzeugen. Bis auf die Passagen im ersten und im siebten Kapitel sind die Darstellungen der Entwicklungen in beiden deutschen Staaten doch recht zusammenhangslos geblieben. Das hängt natürlich auch damit zusammen, dass das Öffentliche Recht in beiden deutschen Staaten sich weitgehend unabhängig voneinander entwickelte. Jedenfalls fehlt im Buch der wichtige ,innerdeutsche Vergleich“. Auch wenn es gewiss schwer ist, hier einen gemeinsamen Vergleichsmaßstab zu finden, wäre dort doch manches Interessantes zu vermelden gewesen: In beiden deutschen Staaten gab es zum Beispiel den Trend zur Spezialisierung durch öffentlich-rechtliche Sondergebiete. Wichtige Fragen könnten sich stellen: Warum gab es in der DDR einen Umweltschutzartikel in der Verfassung längst bevor der Artikel 20a GG im Jahre 1994 geschaffen wurde? Warum gelang es in der DDR bereits 1970 eine erste - freilich recht allgemeine Umweltrechtskodifikation (LKG) zu schaffen, wo doch das UGB-Vorhaben viele Jahre später in der Bundesrepublik Deutschland scheiterte? Wie sah die typische Karriere eines Juraprofessors in der DDR einerseits und in Westdeutschland andererseits aus? Wer durfte Jura studieren, wie stand es mit der Studenten/ProfessorenRelation, wie waren die Berufschancen für Studienabsolventen, wie sah es mit staatlichen Projektförderungen der Rechtswissenschaft aus? Zusammengefasst: Eine 
wirklich vergleichende Wissenschaftsgeschichte des öffentlichen Rechts in beiden deutschen Staaten bleibt somit - auch angesichts des Buches von S. - ein Desiderat für die Zukunft.

Um der thematischen Betrachtung des Buches Herr zu werden, greift S. zu verschiedenen komplexitätsreduzierenden Vorgehensweisen. Er beschränkt seine Quellen im wesentlichen auf Gesetze und auf Entscheidungen des BVerfG, auf Personalverzeichnisse der Fakultäten, öffentlich-rechtliche Handbücher, Lehrbücher und Kommentare sowie auf die Vorträge bei den Staatsrechtslehrertagungen, wobei doch der relative - Bedeutungsverlust dieser Vorträge in der Publikationsflut der letzten Jahrzehnte längst unverkennbar ist. Judikate der Verwaltungsgerichtsbarkeit, Zeitschriftenaufsätze sowie vor allem Monographien einschließlich veröffentlichter Rechtsgutachten bleiben regelmäßig unberücksichtigt. Das ist aus Kapazitätsgründen zwar verständlich, aber wissenschaftlich doch bedauerlich. Gerade die Monographien bringen die Wissenschaft entscheidend voran. Noch schwerwiegender ist die weitgehende Ausblendung der Sondergebiete des Besonderen Verwaltungsrechts, insbesondere auch der hierzu verfassten Literatur. Das Verwaltungsrecht in der Bundesrepublik Deutschland wird ja gegenwärtig faktisch sehr viel stärker durch Fortentwicklung im Besonderen Verwaltungsrecht als im Allgemeinen Verwaltungsrecht bewegt.

Oftmals hat man den Eindruck, das Buch sei wie eine Collage interessanter - vereinzelt möglicherweise auch aus Vorveröffentlichungen hervorgegangener - Einzelbeiträge entstanden (z.B. im vierten Kapitel), die dann unter hinreichend offenen Gesamtüberschriften zusammengefasst wurden. Auch durch diese Technik zusammengefügter Einzelbeiträge kommt es zu vielen überflüssigen Redundanzen im Buch. Insgesamt scheint $S$. dann doch die Kraft zu einer konzisen, systematischen und die Einzelbeiträge wirklich verschmelzenden Darstellung der Geschichte des öffentlichen Rechts zwischen 1945 und 1990 teilweise ausgegangen zu sein.

Deswegen bietet das Buch - wie erwähnt - auch keine strenge chronologische Darstellung, sondern springt hinsichtlich der Berichtszeiten immer wieder hin und her. Dies wiegt schwerer als mancher Flüchtigkeitsfehler (z.B. das bereits angesprochene Fehlen einiger Fakultätsmitglieder und einzelner juristischer Zeitschriften oder falsche Gliederungspunkte (z.B. 578)).

S. bemüht sich - bei aller Kürze - stets um eine faire Beurteilung. Bei manchen Urteilen fragt man sich freilich, welche eigenen fachlichen Vorarbeiten eigentlich die jeweiligen Beurteilungen von S. (etwa im Völkerrecht) legitimieren. Entsprechendes gilt für manche Berichte und Urteile im Besonderen Verwaltungsrecht, wo es bisweilen zu Ungenauigkeiten und verkürzenden Darstellungen kommt. Die Namensaufzählungen vor allem bei den Literaturberichten sind bisweilen ermüdend. Auch 
hier wäre weniger eher mehr gewesen. Was die Zukunftsprognosen von S. vor allem im Ausblick betrifft, sind diese nur wenig wissenschaftlich belegt (und wahrscheinlich auch nicht belegbar). Zu erwähnen bleibt noch, dass S. bei aller deutlichen Entschiedenheit gegenüber den grundlegenden politischen Missständen in der DDR bisweilen erstaunliche Milde im Hinblick auf die DDR-Rechtswissenschaft erkennen lässt, die eben auch Handlanger einer teilweise menschenverachtenden Diktatur war.

Lücken bleiben: Die vielen Grundgesetzänderungen nach 1949 werden kaum berücksichtigt. Eine Betrachtung der Finanzverfassung findet praktisch nicht statt. Der Hauptstadtstreit nach der Wiedervereinigung (Berlin/Bonn) bleibt unerwähnt. Der Einfluss der Wissenschaftsakademien und der Wissenschaftsorganisationen (z.B. Wissenschaftsrat) bzw. der großen Forschungseinrichtungen (wie z.B. Wissenschaftskolleg, WZB) für die Rechtswissenschaft wird nicht erörtert. Privatuniversitäten und Fachhochschulen werden vernachlässigt. Unprofessionell und eher umgangssprachlich ist die sich durch das ganze Buch ziehende Bezeichnung der Bundesrepublik Deutschland als „Bundesrepublik“. Zudem setzt S. fast immer „Frankfurt“ mit Frankfurt/Main gleich und vernachlässigt insoweit Frankfurt/Oder.

4. Insgesamt: Beim Berlin-Marathon - und anderswo - wird jeder Läufer bejubelt, der es bis ins Ziel geschafft hat, der Erste, aber eben auch der Letzte. S. ist bisher der einzige, der sich auf den Marathon einer Gesamtdarstellung der Geschichte des deutschen öffentlichen Rechts bzw. der Wissenschaft des deutschen Öffentlichen Rechts der letzten vier Jahrhunderte gemacht und ihn durchstanden hat. Er ist damit automatisch der Erste - notwendigerweise aber zugleich auch der Letzte. Das Gesamtwerk ist eben auf seinem Gebiet (bislang) Anfang und Ende. Beifall? Ja, trotz aller Mängel: Beifall! 